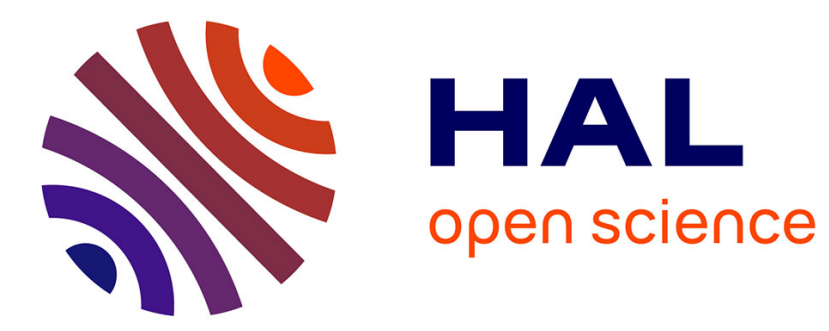

\title{
Conversion from discharge to gate opening for the control of irrigation canals
}

\author{
X. Litrico, P.O. Malaterre, J.P. Baume, J. Ribot Bruno
}

\section{To cite this version:}

X. Litrico, P.O. Malaterre, J.P. Baume, J. Ribot Bruno. Conversion from discharge to gate opening for the control of irrigation canals. Journal of Irrigation and Drainage Engineering, 2008, 134 (3), p. 305 - p. 314. 10.1061/(ASCE)0733-9437(2008)134:3(305) . hal-00453785

\section{HAL Id: hal-00453785 \\ https://hal.science/hal-00453785}

Submitted on 5 Feb 2010

HAL is a multi-disciplinary open access archive for the deposit and dissemination of scientific research documents, whether they are published or not. The documents may come from teaching and research institutions in France or abroad, or from public or private research centers.
L'archive ouverte pluridisciplinaire HAL, est destinée au dépôt et à la diffusion de documents scientifiques de niveau recherche, publiés ou non, émanant des établissements d'enseignement et de recherche français ou étrangers, des laboratoires publics ou privés. 


\title{
Conversion from Discharge to Gate Opening for the Control of Irrigation Canals
}

\author{
X. Litrico, P.-O. Malaterreł J.-P. Baume $\ddagger$ J. Ribot-Bruno ${ }^{\S}$ \\ Final version of paper IRENG-07-5189 accepted for publication in the Journal of \\ Irrigation and Drainage Engineering
}

\begin{abstract}
The paper reviews several methods to convert discharge into gate opening. A control algorithm for one or several reaches of an irrigation canal sometimes uses a discharge as the control action variable even though the device to be manipulated is a gate or a weir. In this case a slave controller has to convert the discharge into a gate opening or a sill elevation in case of a weir. This is usually done by inverting the static relation between discharge and gate opening. An improved method can be based on the characteristics theory to estimate the water levels deviations. However, both methods underestimate the gate opening deviations required to deliver a desired discharge deviation, because water levels vary continuously along time when the gate is operated. The paper proposes a method to take account of this dynamic behavior of the pool-gate interaction by using a simple linear model for the pools dynamics, the IDZ model. The proposed method enables to better estimate the gate opening necessary to get a desired average discharge. The method is evaluated in simulation and on a gate of the Gignac Canal, located in the South of France. A dimensionless analysis of the problem is finally performed to evaluate the methods' applicability.
\end{abstract}

\section{Introduction}

In the design of controllers for irrigation canals, it is well-known that using the discharge as the control variable enables to partially decouple the pools (Malaterre, 1995; Schuurmans, 1997; Malaterre et al., 1998; Malaterre and Baume, 1999). With such a choice as control variable, one needs to use a master-slave structure, where the master controller computes a discharge that is delivered by a local slave controller operating the gate. The functioning of this local slave controller is rarely mentioned in scientific publications, because it appears at first sight as a simple problem. Indeed, if the water levels upstream and downstream of the gate are measured, it seems sufficient to invert the gate discharge equation in order to compute the required gate opening to let a desired discharge flow through the gate. However, this is only

\footnotetext{
${ }^{*}$ Researcher, Unité Mixte de Recherche, Gestion de l'Eau, Acteurs, Usages, Cemagref, B.P. 5095, 34196 Montpellier Cedex 5, France. e-mail: xavier.litrico@cemagref.fr

${ }^{\dagger}$ Researcher, Unité Mixte de Recherche, Gestion de l'Eau, Acteurs, Usages, Cemagref, B.P. 5095, 34196 Montpellier Cedex 5, France. e-mail: pierre-olivier.malaterre@cemagref.fr

${ }^{\ddagger}$ Hydraulic Engineer, Unité Mixte de Recherche, Gestion de l’Eau, Acteurs, Usages, Cemagref, B.P. 5095, 34196 Montpellier Cedex 5, France. e-mail: jean-pierre.baume@cemagref.fr

${ }^{\S}$ Assistant Engineer, Unité Mixte de Recherche, Gestion de l'Eau, Acteurs, Usages, Cemagref, B.P. 5095, 34196 Montpellier Cedex 5, France. e-mail: jose.ribot-bruno@cemagref.fr
} 
true if it is possible to measure the water levels and to operate the gate continuously along time. For computer controlled system, i.e. in the vast majority of cases, this is not possible, because the controller only operates at given sampling time steps. It may be objected that the local slave controller used to manipulate the gate is usually designed to operate at a smaller time step than the master controller which controls the discharge. However, even if this is the case, the gates motors have mechanical constraints which limit the possible number of operations per hour. As an example, some large gates may not be operated more than a few times an hour, which directly limits the operation of a local slave controller. A limited number of operations of the slave local controller is also advisable to limit the power consumption of the control system.

Therefore, the 'natural' solution to the discharge-gate opening conversion (i.e. invert the gate equation continuously along time or with a very small time step, say a few seconds) is usually not applicable in this case. The slave controller is a priori not able to provide the required discharge as specified by the master controller. Indeed, when the gate is operated, the water levels immediately upstream and downstream of the gate change instantaneously, and therefore modify the discharge, which is then no longer equal to the desired one.

Then a static inversion may not be an efficient method. To our knowledge, despite its poor performance as demonstrated in this paper, the static method is the only one used for the moment in the field for controllers with a master-slave structure.

To overcome this limitation, De Leon Mojarro (1986) proposed to use the characteristics approximation to predict the water levels variations due to a discharge change at a gate. This solution improves the static inversion method by taking into account the initial deviations in water levels due to the gate operation as calculated using the characteristics theory. But the model remains quasi-static, because the water levels and the discharge are supposed to stay constant after the gate movement, which is not true.

Because it is not possible to ensure a constant discharge through the gate during the whole sampling period $T_{s}$, we suggest to calculate the gate opening deviation so as to ensure that the average discharge flowing through the gate during $T_{s}$ corresponds to the desired one.

We propose in this paper a way to take account of the full dynamic behavior of a canal when a gate is operated. The proposed method uses a simplified linear dynamic model for each pool, the IDZ model presented by Litrico and Fromion (2004b), which enables to compute a closed-form linear approximation of the discharge flowing through the gate along time. This gives us a way to better approximate the gate opening necessary to get a desired average discharge during the sampling time period $T_{s}$. A nonlinear version of this dynamic method is also proposed to cover for the gate nonlinearities.

The paper is structured as follows:

- The dynamic behavior of gate-channel interaction is first examined, and three approximations are compared for estimating the discharge flowing through a gate after a step gate opening or closing: the static approximation, the one based on the characteristics, and the dynamic one, based on the IDZ model. Both a linear and a nonlinear version of each method are presented.

- The problem of the conversion from discharge to gate opening is then considered, and the three methods are compared in simulation.

- A field experiment is reported where the three methods are compared on a gate of the Gignac Canal, located in the South of France, near Montpellier. 
- A dimensionless linear analysis is performed to get a priori evaluation of the methods' applicability.

\section{Dynamic Behavior of Gate-Channel Interaction}

We consider the hydraulic behavior of two canal pools interconnected with a hydraulic crossstructure, as depicted in Fig. 1 in the case of a gate. The methods developed in the paper are also applicable in the case of a moveable weir, or a more complex structure, provided the discharge flowing through this structure can be estimated via a static nonlinear equation (the discharge equation).

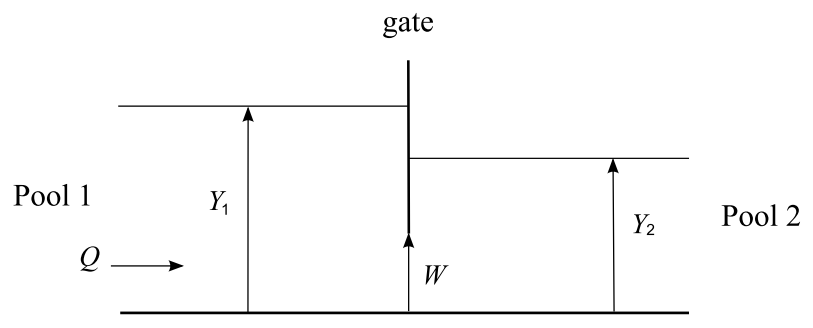

Figure 1: Schematic representation of a gate between two canal pools

Let us denote $Q$ the discharge flowing through the gate, $Y_{1}$ the water level upstream of the gate (i.e. the water level downstream of pool 1), $Y_{2}$ the water level downstream of the gate (i.e. the water level upstream of pool 2) and $W$ the gate opening. The gate equation writes:

$$
Q=f\left(Y_{1}, Y_{2}, W\right)
$$

Typically, for a submerged orifice, the function $f$ is given by :

$$
f\left(Y_{1}, Y_{2}, W\right)=C_{d} \sqrt{2 g} L_{g} W \sqrt{Y_{1}-Y_{2}}
$$

where $C_{d}$ is a discharge coefficient, $L_{g}$ the gate width, and $g=9.81 \mathrm{~ms}^{-2}$ the gravitational acceleration.

In the following, the methods will be developed using a nonlinear and a linear version. The linear approximation is derived by assuming small deviations in gate opening, water levels and discharge, which leads to the linearized gate equation:

$$
q=k_{u} y_{1}-k_{d} y_{2}+k_{w} w
$$

where $q$ is the discharge deviation from reference value $Q, y_{i}$ are the water level deviations from reference values $Y_{i}(i=1,2), w$ is the deviation from the reference gate opening $W$, and coefficients $k_{u},-k_{d}$ and $k_{w}$ are respectively obtained by differentiating function $f$ of Eq. (1) with respect to its first, second and third arguments, respectively.

Let us now suppose that the gate is suddenly opened or closed at a time instant $t=0$, changing the opening from $W$ to $W+w_{0}$. We compare below three different approximations for evaluating the discharge flowing through the gate after this operation. 


\section{Static Approximation}

In the static approximation, it is assumed that the water levels $Y_{1}$ and $Y_{2}$ do not vary along time during the sampling period $T_{s}$. The nonlinear static approximation of the discharge deviation, denoted $q_{s}^{n l}$ is therefore:

$$
q_{s}^{n l}=f\left(Y_{1}, Y_{2}, W+w_{0}\right)-f\left(Y_{1}, Y_{2}, W\right)
$$

The linearized version of Eq. (3) leads to the linear static approximation of the discharge deviation, denoted $q_{s}^{l}$ :

$$
q_{s}^{l}=k_{w} w_{0}
$$

which means that change in flow is proportional to change in gate opening. This is a static approximation because the water levels are supposed to stay constant.

\section{Approximation with the Characteristics}

This method uses the characteristics for zero slope rectangular frictionless channels to approximate the effect of the gate opening on the water levels. Let us denote $q_{c}^{v}$ the discharge deviation resulting from the gate opening, with $v=l$ for the linear version, and $v=n l$ for the nonlinear version.

The deviations in water levels due to this discharge deviation $q_{c}^{v}$ can be approximated by the characteristics method for a horizontal rectangular frictionless channel, leading to (De Leon Mojarro, 1986):

$$
\begin{aligned}
& y_{1 c}^{v}=-\frac{q_{c}^{v}}{T_{1}\left(C_{1}-V_{1}\right)} \\
& y_{2 c}^{v}=\frac{q_{c}^{v}}{T_{2}\left(C_{2}+V_{2}\right)},
\end{aligned}
$$

where $C_{i}, V_{i}, T_{i}$ are respectively the celerity, the velocity and the top width in pool $i(i=1,2)$.

For the nonlinear characteristics approximation, the resulting discharge deviation $q_{c}^{n l}$ is obtained as the solution of the equation:

$$
q_{c}^{n l}=f\left(Y_{1}+y_{1 c}^{n l}, Y_{2}+y_{2 c}^{n l}, W+w_{0}\right)-f\left(Y_{1}, Y_{2}, W\right),
$$

which is implicit, because $y_{1 c}^{n l}$ and $y_{2 c}^{n l}$ depend on the unknown $q_{c}^{n l}$.

For the linear characteristics approximation, an explicit solution can be found. The linearized gate equation (2) is rewritten for convenience:

$$
q_{c}^{l}=k_{u} y_{1 c}^{l}-k_{d} y_{2 c}^{l}+k_{w} w_{0} .
$$

Then, combining Eqs. (5-6) with Eq. (8) yields $q_{c}^{l}$ given by:

$$
q_{c}^{l}=\frac{k_{w} w_{0}}{1+\frac{k_{u}}{T_{1}\left(C_{1}-V_{1}\right)}+\frac{k_{d}}{T_{2}\left(C_{2}+V_{2}\right)}} .
$$

The nonlinear and linear characteristics approximations are quasi-static, because the water levels are supposed to change instantaneously with the gate opening at time $t$, and then to stay constant.

Let us note that in the case of subcritical flow in pool $1\left(C_{1}>V_{1}\right)$, the discharge deviation $q_{c}^{v}$ computed with the linear or nonlinear characteristics approximation is always smaller in absolute value than the discharge deviation $q_{s}^{v}$ computed with the static approximation: $\left|q_{c}^{v}\right|<$ $\left|q_{s}^{v}\right|$. 


\section{Dynamic Approximation with the IDZ model}

We now propose a new approach based on linear dynamic models for predicting the water levels deviations in each pool. These linear dynamic models are derived from linearized Saint-Venant equations (Litrico and Fromion, 2004a) and are expressed as transfer functions in the Laplace complex variable $s$. The Laplace transform of a given function $f(t)$ is denoted $\hat{f}(s)$.

\section{Interconnection with Linear Models}

We first give the general expression of the interconnection of the pools with the intermediate gate. We denote $q_{d}^{l}(t)$ the linear version of the dynamic approximation of the discharge deviation. Pool 1 is modelled by the linear dynamic equation:

$$
\hat{y}_{1}(s)=g_{1}(s) \hat{q}_{d}^{l}(s)
$$

And pool 2 is modelled by a similar equation:

$$
\hat{y}_{2}(s)=g_{2}(s) \hat{q}_{d}^{l}(s)
$$

where $g_{i}(s)$ denote the input-output transfer functions of linearized Saint-Venant equations, relating the discharge to the water levels of pool $i(i=1,2)$. See Litrico and Fromion (2004a) for detailed expressions of transfer functions $g_{1}(s)$ and $g_{2}(s)$.

Combining the equations (10-11) with the linearized gate equation (2) leads to the linear equation describing the dynamic behavior of the gate discharge as a function of the gate opening:

$$
\hat{q}_{d}^{l}(s)=\frac{k_{w}}{1-k_{u} g_{1}(s)+k_{d} g_{2}(s)} \hat{w}(s)
$$

This equation provides a dynamic approximation of the discharge flowing through a gate when the gate is operated. It can be computed numerically using accurate approximations of transfer functions $g_{1}(s)$ and $g_{2}(s)$. Moreover, it is possible to derive an analytical solution for step gate movement (opening or closing) using a simple approximation for both transfer functions, the IDZ model approximation proposed by Litrico and Fromion (2004b).

\section{Analytical Solution with the IDZ Model}

As demonstrated hereafter, using the IDZ model approximation for $g_{1}(s)$ and $g_{2}(s)$ can lead to an analytical expression of the discharge $q_{d}^{l}(t)$ for a step opening. The IDZ model gives the following approximations of transfer functions $g_{2}(s)$ and $g_{1}(s)$ (Litrico and Fromion, 2004b):

$$
\begin{aligned}
& g_{1}(s)=-\frac{a_{1} s+b_{1}}{s} \\
& g_{2}(s)=\frac{a_{2} s+b_{2}}{s}
\end{aligned}
$$

where $a_{1}, a_{2}$ are the high frequency gains in $\mathrm{sm}^{-2}$ and $b_{1}, b_{2}$ are the integrator gains in $\mathrm{m}^{-2}$.

Using these approximations in Eq. (12) gives:

$$
\hat{q}_{d}^{l}(s)=\frac{k_{w} s}{a s+b} \hat{w}(s)
$$

with

$$
a=1+k_{u} a_{1}+k_{d} a_{2}
$$


and

$$
b=k_{u} b_{1}+k_{d} b_{2}
$$

The inverse Laplace transform of the step response (input $\hat{w}(s)=w_{0} / s$ ) of this first order linear dynamic system is given by (see appendix for details):

$$
q_{d}^{l}(t)=\left(\frac{k_{w}}{a} w_{0}+q_{0}\right) e^{-\frac{b}{a} t}
$$

which is the dynamic linear model approximation $q_{d}^{l}(t)$ of the discharge flowing through the gate after a step gate operation of amplitude $w_{0}$ at time $t=0$, with an initial condition $q_{0}$, corresponding to the deviation between the actual discharge and the discharge used for linearization.

This model also gives analytical expressions for the variations of water levels. Using Eqs. (10-11), the water levels deviations are given by:

$$
\begin{aligned}
& y_{1}(t)=-a_{1} q_{d}^{l}(t)-b_{1} \int_{0}^{t} q_{d}^{l}(v) d v \\
& y_{2}(t)=a_{2} q_{d}^{l}(t)+b_{2} \int_{0}^{t} q_{d}^{l}(v) d v
\end{aligned}
$$

Finally, we obtain:

$$
\begin{aligned}
& y_{1}(t)=-a_{1}\left(\frac{k_{w}}{a} w_{0}+q_{0}\right)\left[\frac{a b_{1}}{b a_{1}}+\left(1-\frac{a b_{1}}{b a_{1}}\right) e^{-\frac{b}{a} t}\right] \\
& y_{2}(t)=a_{2}\left(\frac{k_{w}}{a} w_{0}+q_{0}\right)\left[\frac{a b_{2}}{b a_{2}}+\left(1-\frac{a b_{2}}{b a_{2}}\right) e^{-\frac{b}{a} t}\right]
\end{aligned}
$$

\section{Link with the Characteristics Method}

It is easy to show that the linear characteristics method solution corresponds to the high frequency gains component of the linear dynamic method. In this case, only parameter $a_{i}$ $(i=1,2)$ is used in the IDZ model approximation $(13-14)$ and parameter $b_{i}(i=1,2)$ is assumed to be null.

For a canal pool at uniform flow, the parameters $a_{1}$ and $a_{2}$ are obtained by the following equations (Litrico and Fromion, 2004b):

$$
\begin{aligned}
& a_{1}=\frac{1}{T_{1}\left(C_{1}-V_{1}\right)} \sqrt{\frac{\left(\frac{1-F_{1}}{1+F_{1}}\right)^{2}+e^{\alpha_{1} X_{1}}}{1+e^{\alpha_{1} X_{1}}}} \\
& a_{2}=\frac{1}{T_{2}\left(C_{2}+V_{2}\right)} \sqrt{\frac{\left(\frac{1+F_{2}}{1-F_{2}}\right)^{2}+e^{\alpha_{2} X_{2}}}{1+e^{\alpha_{2} X_{2}}}}
\end{aligned}
$$

where $\alpha_{i}$ is a positive constant linked to the pool characteristics and $X_{i}$ is the length of pool $i$.

When $X_{i}(i=1,2)$ tends towards $+\infty$, the square roots in Eqs. (23-24) tend towards 1 , and the high frequency gains of the IDZ model tend towards the gains computed with the characteristics method in Eqs. (5-6):

$$
\begin{array}{ll}
a_{1} \rightarrow \frac{1}{T_{1}\left(C_{1}-V_{1}\right)} \quad ; \quad b_{1}=0 \\
a_{2} \rightarrow \frac{1}{T_{2}\left(C_{2}+V_{2}\right)} \quad ; \quad b_{2}=0 .
\end{array}
$$


Therefore, the linear characteristics method corresponds to the linear dynamic method with IDZ models where only the high frequency elements are considered, i.e. where the integrators are not taken into account $\left(b_{1}=b_{2}=0\right)$.

Let us note that the time behavior is consistent the frequency domain analysis. Indeed, the high frequency behavior corresponds to the initial value of $q_{d}^{l}(t)$ at $t=0$. According to Eq. (18), for canals such that Eqs. (25-26) are fulfilled, and for a zero initial condition $\left(q_{0}=0\right)$, we have:

$$
q_{d}^{l}(0)=\frac{k_{w} w_{0}}{a}=q_{c}^{l}
$$

where $q_{c}^{l}$ is given by Eq. (9).

We recover the fact that the high frequency dynamic approximation and the characteristics one yield the same discharge deviations.

\section{Nonlinear Dynamic Method}

It is unfortunately not possible to explicitly compute a nonlinear version of the dynamic method. This would mean solving the complete Saint-Venant equations coupled with the gate equation. A simplified problem consists in solving the nonlinear dynamic interaction between linear IDZ models of the pools and the nonlinear model of the gate. Even this problem is complex to solve directly, because the resulting equation is an algebraic nonlinear differential equation.

We denote by $T_{s}$ the sampling time interval. Our objective is to better approximate the discharge when hydraulic conditions deviate from the ones used for linearization, e.g. the ones encountered $T_{s}$ seconds after a gate movement.

We therefore propose an approximate nonlinear version of the dynamic method that uses two linear approximations: one at the beginning and one at the end of the sampling time interval $\left[0, T_{s}\right]$.

The first one is given by Eq. (18), and the second one is given by the same equation, but where the parameters $a$ and $b$ are computed with the new gate linearized parameters $k_{u}, k_{d}$, $k_{w}$, corresponding to the new hydraulic state. This hydraulic state is based on the initial linear simulation, using Eqs. (21-22) to evaluate the water levels deviations.

Finally, the nonlinear dynamic approximation of the discharge deviation is given by:

$$
q_{d}^{n l}(t)=\frac{1}{2}\left(q_{d, 0}^{l}(t)+q_{d, T_{s}}^{l}(t)\right),
$$

where $q_{d, 0}^{l}(t)$ and $q_{d, T_{s}}^{l}(t)$ are given by Eq. (18) with parameters $a$ and $b$ respectively computed for the hydraulic state at time $t=0$ and $t=T_{s}$.

\section{Comparison of the Responses to a Step Gate Opening}

The three approximations are compared to the exact solution obtained from a full nonlinear simulation with software SIC (Simulation of Irrigation Canals) developed by Cemagref, Montpellier, France (Malaterre, 2006). SIC uses a semi-implicit Preissmann scheme to solve Saint-Venant equations, and includes the nonlinear hydraulic structures equations in the resolution.

For illustration purposes, a hypothetical canal is simulated in SIC. This canal, corresponding to a part of the Cemagref benchmark canal N.1 (Baume and Sau, 1997), has two identical pools separated by a sluice gate. Each pool is $3000 \mathrm{~m}$ long, with a trapezoidal shape, a bottom width of $7 \mathrm{~m}$, a side slope of 1.5, a bottom slope of 0.0001 , and a Manning coefficient of 0.02 . A 
bottom step of $0.24 \mathrm{~m}$ is present between the pools at the gate location. The canal pools are supposed to be at normal depth $Y=2.12 \mathrm{~m}$ for the discharge $Q=14 \mathrm{~m}^{3} \mathrm{~s}^{-1}$.

These two identical canal pools are interconnected with a gate, of width $L_{g}=10.18 \mathrm{~m}$, discharge coefficient $C_{d}=0.82$, gate opening $W=0.654 \mathrm{~m}$. This leads to the following linearized gate coefficients: $k_{u}=22.12 \mathrm{~m}^{2} \mathrm{~s}^{-1}, k_{d}=26.74 \mathrm{~m}^{2} \mathrm{~s}^{-1}$ and $k_{w}=35.41 \mathrm{~m}^{2} \mathrm{~s}^{-1}$.

The simulation in SIC is done with a time step of $\Delta t=60 \mathrm{~s}$, a spatial sampling of $\Delta x=200$ $\mathrm{m}$, which leads to a Courant number close to one. This ensures a correct simulation of oscillating waves.

\section{Discharge Approximations}

We first evaluate the discharge approximations with the three methods for a step gate opening of $0.03 \mathrm{~m}$.

\section{Static Method}

Following Eq. (4), the static method gives an estimate of the discharge deviation equal to $q_{s}^{n l}=3.60 \mathrm{~m}^{3} \mathrm{~s}^{-1}$ in the nonlinear case, and $q_{s}^{l}=3.54 \mathrm{~m}^{3} \mathrm{~s}^{-1}$ in the linear case.

\section{Characteristic Method}

For the characteristics method, we have the following values for the velocities, the celerities and the top widths of each pool: $V_{1}=V_{2}=0.74 \mathrm{~ms}^{-1}, C_{1}=C_{2}=3.82 \mathrm{~ms}^{-1}$ and $T_{1}=T_{2}=12.76$ $\mathrm{m}$. The nonlinear characteristics method Eq. (7) provides the following estimate of discharge deviation $q_{c}^{n l}=1.488 \mathrm{~m}^{3} \mathrm{~s}^{-1}$, and the linear characteristics method of Eq. (9) gives $q_{c}^{l}=1.559$ $\mathrm{m}^{3} \mathrm{~s}^{-1}$.

\section{Dynamic Method}

Using the canals parameters, the IDZ model parameters are given by $a_{1}=0.022 \mathrm{sm}^{-2}, b_{1}=$ $3.22 \times 10^{-5} \mathrm{~m}^{-2}, a_{2}=0.017 \mathrm{sm}^{-2}, b_{2}=1.89 \times 10^{-5} \mathrm{~m}^{-2}$.

Following Eqs. (16-17) the parameters for the linear dynamic method are given by $a=1.936$ and $b=1.2 \times 10^{-3} \mathrm{~s}^{-1}$. The discharge deviation for the linear dynamic method is then given by Eq. (18).

For the nonlinear dynamic method, we choose a time interval of $T_{s}=1 \mathrm{~h}$, and we compute the water levels deviations predicted by the linear model: $y_{1}\left(T_{s}\right)=-0.06 \mathrm{~m}$ and $y_{2}\left(T_{s}\right)=0.038$ $\mathrm{m}$. The new linearized gate coefficients are then given by $k_{u}=53.17 \mathrm{~m}^{2} \mathrm{~s}^{-1}, k_{d}=56.54 \mathrm{~m}^{2} \mathrm{~s}^{-1}$ and $k_{w}=20.45 \mathrm{~m}^{2} \mathrm{~s}^{-1}$. This leads to $a_{T_{s}}=3.119$ and $b_{T_{s}}=2.8 \times 10^{-3} \mathrm{~s}^{-1}$.

The final discharge approximation obtained with the nonlinear version of the dynamic method is given by Eq. (28).

\section{Comparison of Results}

Figure 2 depicts the discharge along time after this step opening, comparing the result of the nonlinear simulation using SIC software to the three methods described in the previous sections.

The oscillation observed at the beginning of the simulation is due to the reflection of propagating waves on the boundary conditions. Indeed, this canal is rather flat, and presents an oscillatory behavior in high frequencies (Litrico and Fromion, 2004a). 


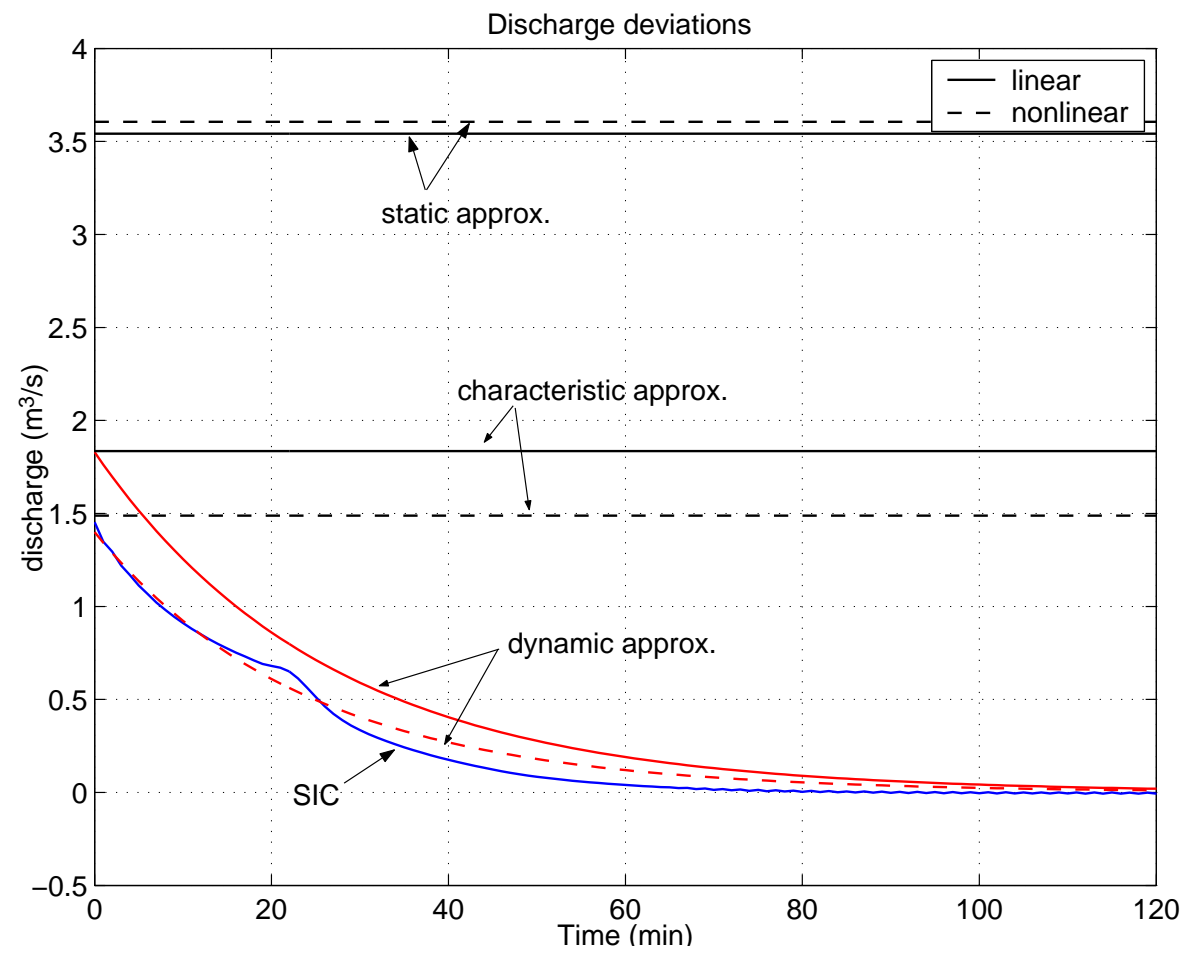

Figure 2: Time variation of discharge deviation $q(t)$ for $w_{0}=0.10 \mathrm{~m}$. Comparison with linear and nonlinear versions of dynamic, characteristic and static approximations.

It is clear that the linear and nonlinear versions of the dynamic method lead to a very good approximation of the discharge flowing through the gate along time. The characteristics method gives a good approximation of the peak discharge just after the gate opening. The static method gives the worst approximation. The nonlinear versions usually improve the discharge estimation compared to the linear ones, but it appears that the main source of discrepancy comes from the fact that static and characteristics methods neglect the dynamic behavior of the discharge.

\section{Effect of the Sampling Period $T_{s}$}

Based on the step response results of Fig. 2, we now evaluate the precision of the volume flowing through the gate according to the various approximations. The initial control objective is to deliver a desired discharge during the sampling period $T_{s}$. Because it is not possible to keep the discharge constant between two gate operations, we consider an alternative objective, which is to deliver a desired volume during the sampling period.

To evaluate the precision of the volume estimation of the three methods, we compare the volume effectively flowing through the gate (computed with SIC model) to the one predicted by each method. The relative error depends on the chosen sampling time, and is defined by:

$$
e_{s, c, d}^{v}\left(T_{s}\right)=100 \times \frac{\int_{0}^{T_{s}} q_{s, c, d}^{v}(t) d t-\int_{0}^{T_{s}} q_{S I C}(t) d t}{\int_{0}^{T_{s}} q_{S I C}(t) d t}
$$

where the subscript $s, c, d$ refer to the static, characteristic, and dynamic method, respectively, 
the superscript $v$ refers to the linear or nonlinear version of the method, and $q_{S I C}(t)$ denotes the discharge deviation computed using SIC model.

Figure 3 depicts the relative error between the deviation in delivered volume computed by SIC, and computed by the various approximations as a function of $T_{s}$. It appears clearly that when the sampling period increases, the characteristics and the static methods give an error which increases linearly with the sampling period. On the contrary, the dynamic method gives an error which is almost constant, even for large sampling periods $T_{s}$.

The static method gives a relative error which is always higher than $150 \%$, and the characteristic method is better, but its relative error increases with the sampling period, and becomes larger than $50 \%$ when the sampling time is larger than 18 minutes. The linear dynamic method leads to an error of $50 \%$ when $T_{s}=1 \mathrm{~h}$, but this error is not very sensitive to the increase of $T_{s}$ compared to the other two methods. The nonlinear dynamic method has an error always lower than $10 \%$.

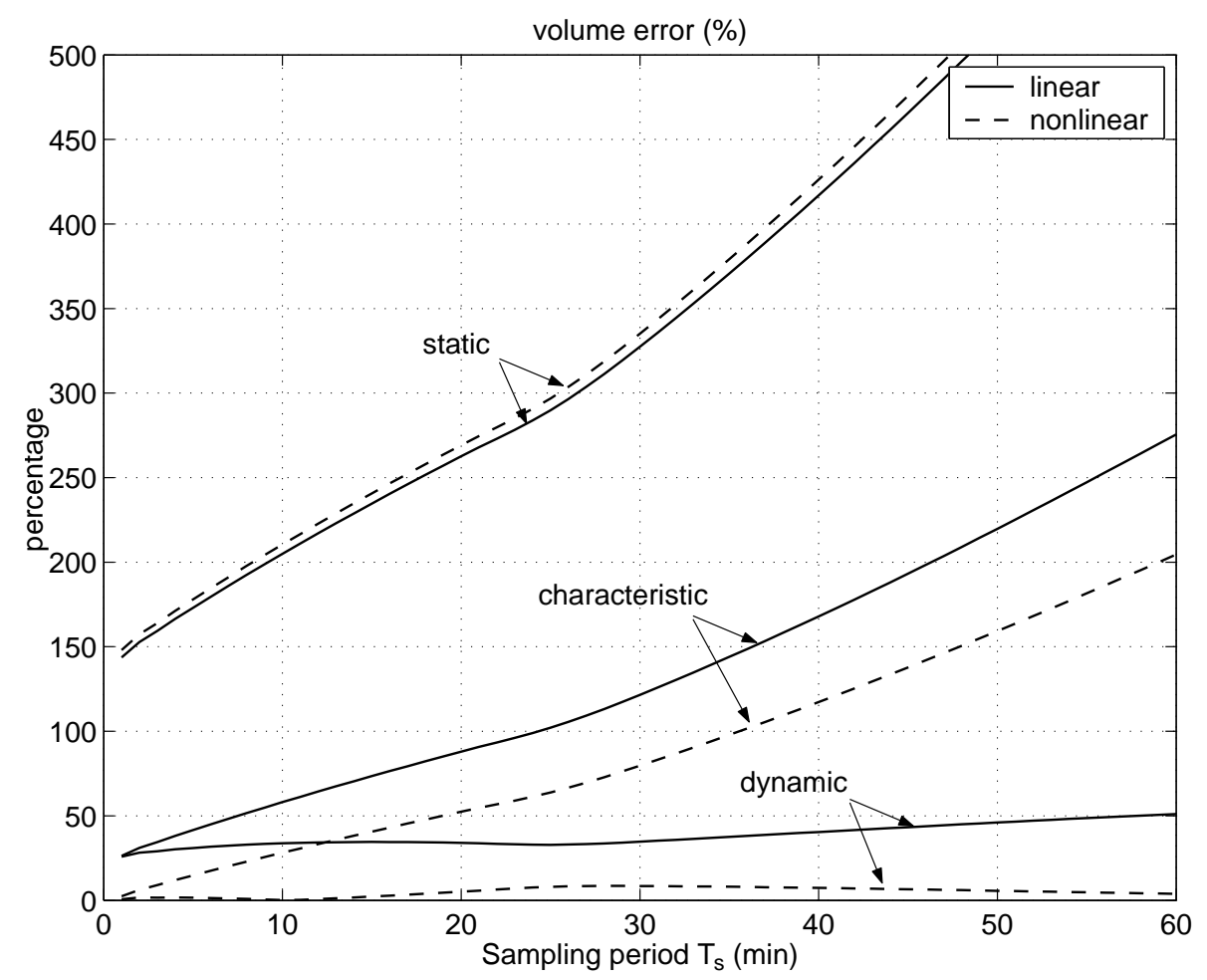

Figure 3: Percentage error between delivered volumes. Comparison of linear and nonlinear versions of static, characteristic and dynamic approximations.

This confirms the fact that an accurate method for discharge-gate opening conversion may improve the performance of computer controlled irrigation canals.

\section{Methods for Discharge/Gate Opening Conversion}

Based on the previous analysis, we derive a new method for discharge/gate opening conversion. The objective is now to compute the gate opening deviation $w$ so that the discharge deviation is equal, if possible, to the desired discharge denoted $\bar{q}$ during the sampling period $T_{s}$. 


\section{Static Conversion}

In the static conversion method, the calculation of the gate position is made assuming that the water levels on both sides of the gate are staying constant, equal to their initial value at the time of the gate operation.

Therefore, in the nonlinear version, the gate opening deviation $w_{s}^{n l}$ is obtained by inverting Eq. (3), which leads to solve the following problem:

$$
\text { find } w_{s}^{n l} \text { such that } \bar{q}=f\left(Y_{1}, Y_{2}, W+w_{s}^{n l}\right)-f\left(Y_{1}, Y_{2}, W\right)
$$

For the linear version, the gate opening deviation $w_{s}^{l}$ to obtain a desired discharge deviation $\bar{q}$ is derived by inverting Eq. (4):

$$
w_{s}^{l}=\frac{\bar{q}}{k_{w}}
$$

\section{Conversion with the Characteristics}

In the characteristics approximation method, the water levels are supposed to instantly move according to the characteristics of each pool. Therefore, for the nonlinear version, the gate opening deviation $w_{c}^{n l}$ is obtained by solving the following problem using Eq. (7):

$$
\text { find } w_{c}^{n l} \text { such that } \bar{q}=f\left(Y_{1}+y_{1 c}^{n l}, Y_{2}+y_{2 c}^{n l}, W+w_{c}^{n l}\right)-f\left(Y_{1}, Y_{2}, W\right)
$$

with $y_{1 c}^{n l}$ and $y_{2 c}^{n l}$ given by Eqs. (5-6) where $q_{c}^{n l}=\bar{q}$.

The linear version leads to an explicit solution because the gate opening deviation $w_{c}^{l}$ is derived by inverting Eq. (9):

$$
w_{c}^{l}=\left(1+\frac{k_{u}}{T_{1}\left(C_{1}-V_{1}\right)}+\frac{k_{d}}{T_{2}\left(V_{2}+C_{2}\right)}\right) \frac{\bar{q}}{k_{w}}
$$

One may show that the gate opening deviation obtained with the characteristics method is larger than the one obtained with the static method in absolute value, in the linear as in the nonlinear case $\left|w_{c}^{v}\right|>\left|w_{s}^{v}\right|$. This is due to the fact that, as seen above, for the same gate opening deviation, the water levels deviations decrease the discharge deviation compared to the static case. Therefore, a larger gate opening deviation is necessary to deliver the same discharge deviation.

\section{Dynamic Conversion}

Using the dynamic linear approximation of the discharge (18), we can compute the volume flowing through the gate after a step operation of $w_{d}^{l}$ :

$$
\begin{aligned}
\int_{0}^{T_{s}} q_{d}^{l}(t) d t & =\left(\frac{k_{w}}{a} w_{d}^{l}+q_{0}\right) \int_{0}^{T_{s}} e^{-\frac{b}{a} t} d t \\
& =\left(\frac{k_{w}}{a} w_{d}^{l}+q_{0}\right) \frac{a}{b}\left(1-e^{-\frac{b}{a} T_{s}}\right)
\end{aligned}
$$

The objective is to compute $w_{d}^{l}$ such that the average delivered discharge over the sampling period $T_{s}$ equals the required discharge $\bar{q}$, i.e.:

$$
\int_{0}^{T_{s}} q_{d}^{l}(t) d t=T_{s} \bar{q}
$$


Combining Eqs. (35-36) and solving for $w_{d}^{l}$ leads to

$$
w_{d}^{l}=\frac{a}{k_{w}}\left[\frac{b T_{s} \bar{q}}{a\left(1-e^{-\frac{b}{a} T_{s}}\right)}-q_{0}\right]
$$

which is the result of the linear version of the dynamic approximation method, computing the gate opening deviation in order to deliver an average discharge $\bar{q}$ during the period $T_{s}$, taking into account the initial discharge deviation $q_{0}$.

In the nonlinear dynamic approximation method, the volume can also be expressed explicitly with Eq. (28):

$$
\int_{0}^{T_{s}} q_{d}^{n l}(t) d t=\frac{1}{2}\left[\left(\frac{k_{w, 0}}{a_{0}} w_{d}^{n l}+q_{0}\right) \beta_{0}+\left(\frac{k_{w, T_{s}}}{a_{T_{s}}} w_{d}^{n l}+q_{0}\right) \beta_{T_{s}}\right]
$$

with $a_{0}$ and $a_{T_{s}}$ denoting the parameter $a$ corresponding to values computed at $t=0$, and at $t=T_{s}$, and parameters $\beta_{0}$ and $\beta_{T_{s}}$ given by:

$$
\begin{aligned}
\beta_{0} & =\frac{1}{p_{0}}\left(e^{p_{0} T_{s}}-1\right) \\
\beta_{T_{s}} & =\frac{1}{p_{T_{s}}}\left(e^{p_{T_{s}} T_{s}}-1\right)
\end{aligned}
$$

where $p_{0}=-b_{0} / a_{0}$ is the pole of the transfer function obtained in Eq. (15) for the initial condition and $p_{T_{s}}=-b_{T_{s}} / a_{T_{s}}$ the pole for the final condition.

The result of the nonlinear version of the dynamic approximation method is then:

$$
w_{d}^{n l}=\frac{2 a_{0} a_{T_{s}}}{k_{w, 0} a_{T_{s}} \beta_{0}+k_{w, T_{s}} a_{0} \beta_{T_{s}}}\left[T_{s} \bar{q}-\frac{\left(\beta_{0}+\beta_{T_{s}}\right)}{2} q_{0}\right]
$$

\section{Effect of the Sampling Period $T_{s}$}

Let us compare the gate opening deviations $\left(w_{s}^{v}, w_{c}^{v}, w_{d}^{v}\right)$ obtained by the three methods for various sampling time steps $T_{s}$. We use the three approximations (both in linear and nonlinear case) to compute the gate opening deviations necessary to deliver a discharge deviation of $1 \mathrm{~m}^{3} \mathrm{~s}^{-1}$ during the sampling period $T_{s}$. The results are depicted in Fig. 4 (note that the linear and nonlinear versions of the static conversion method lead to very close gate openings, which explains why the two lines are difficult to distinguish). The static and the characteristics approximations lead to constant values whatever the size of the sampling period, while the dynamic approximation leads to a larger gate opening deviation when the sampling period increases. Therefore, one should preferably use the dynamic method for large sampling periods, in order to deliver the proper volume.

We now compare the three methods for discharge/gate opening conversion, first in simulation using SIC software, second with a real-life experiment on a gate of the Gignac Canal, located in the South of France, close to Montpellier.

\section{Simulation Results}

The simulations are done on the same system with two identical pools presented above.

We evaluate the ability of the three methods to follow a set-point variation. The required set-point is a step increase in discharge deviation of $0.5 \mathrm{~m}^{3} \mathrm{~s}^{-1}$ during 2 hours. The simulation 


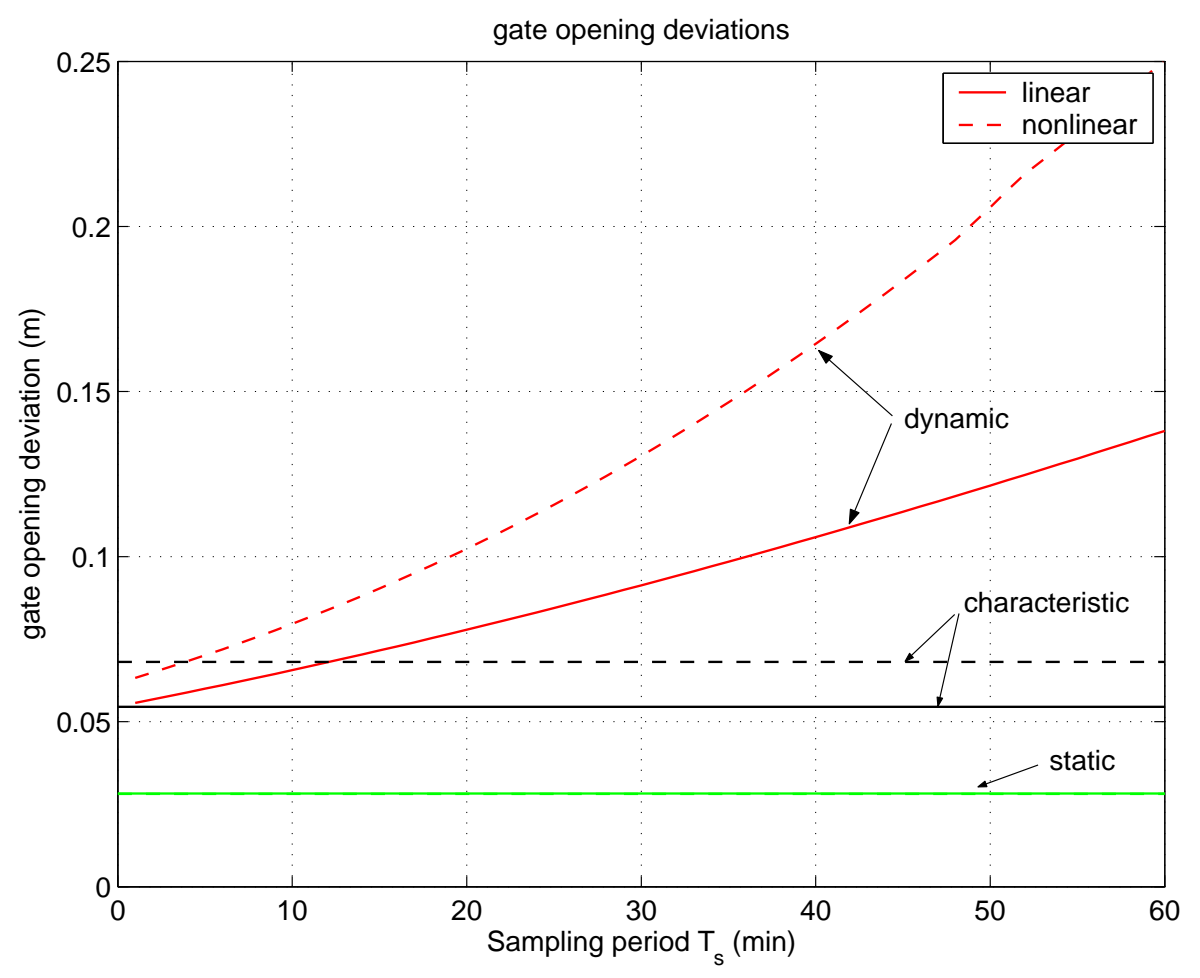

Figure 4: Gate opening deviations to deliver $1 \mathrm{~m}^{3} \mathrm{~s}^{-1}$. Comparison of linear and nonlinear versions of dynamic, characteristic and static approximations for various sampling periods $T_{s}$.

time step is equal to 60 seconds in order to accurately represent the flow dynamics at the gate, and the total simulation time is equal to $10 \mathrm{~h}$.

We compare the three methods (for both linear and non-linear case) for different values of the sampling period $T_{s}: T_{s}=10,20,30$, and 60 minutes. The performance of each method is evaluated by computing the relative error of the delivered volume as defined in Eq. (29). The results are given in table 1 .

Table 1: Percentage error of delivered volume for the set-point following scenario for different values of the sampling time $T_{s}$

\begin{tabular}{c|c|c|c|c|c}
\hline \hline method & version & $T_{s}=10 \mathrm{~min}$ & $T_{s}=20 \mathrm{~min}$ & $T_{s}=30 \mathrm{~min}$ & $T_{s}=60 \mathrm{~min}$ \\
\hline static & linear & $48.1 \%$ & $63.0 \%$ & $70.9 \%$ & $83.5 \%$ \\
& nonlinear & $50.1 \%$ & $64.5 \%$ & $72.0 \%$ & $84.0 \%$ \\
\hline characteristics & linear & $24.8 \%$ & $39.1 \%$ & $47.2 \%$ & $66.0 \%$ \\
& nonlinear & $24.0 \%$ & $37.7 \%$ & $45.5 \%$ & $64.4 \%$ \\
\hline dynamic & linear & $9.2 \%$ & $13.8 \%$ & $15.2 \%$ & $8.0 \%$ \\
& nonlinear & $5.7 \%$ & $6.5 \%$ & $3.4 \%$ & $7.4 \%$ \\
\hline \hline
\end{tabular}

It clearly appears that the dynamic method outperforms the other methods for delivering the required discharge. An interesting feature of the dynamic method is that its performance does not significantly decrease when the sampling time increases. It also appears that, even for small sampling times, both linear and nonlinear versions of the dynamic method leads to 
much better results than the other methods, because it delivers at least $85 \%$ of the desired volume, while the static method always delivers less than $50 \%$ of the desired volume. The characteristics method is in between, with an error ranging from $24 \%$ to $65 \%$ for the considered sampling time periods.

An example of this dynamic behavior is depicted in Fig. 5, where the three methods are compared for $T_{s}=60 \mathrm{~min}$. Due to the dynamic behavior of the discharge, one needs to open the gate much more than predicted by the static or even the characteristics method in order to deliver a given discharge. This is what the dynamic method does, by taking into account a good approximation of upstream and downstream water levels fluctuations.

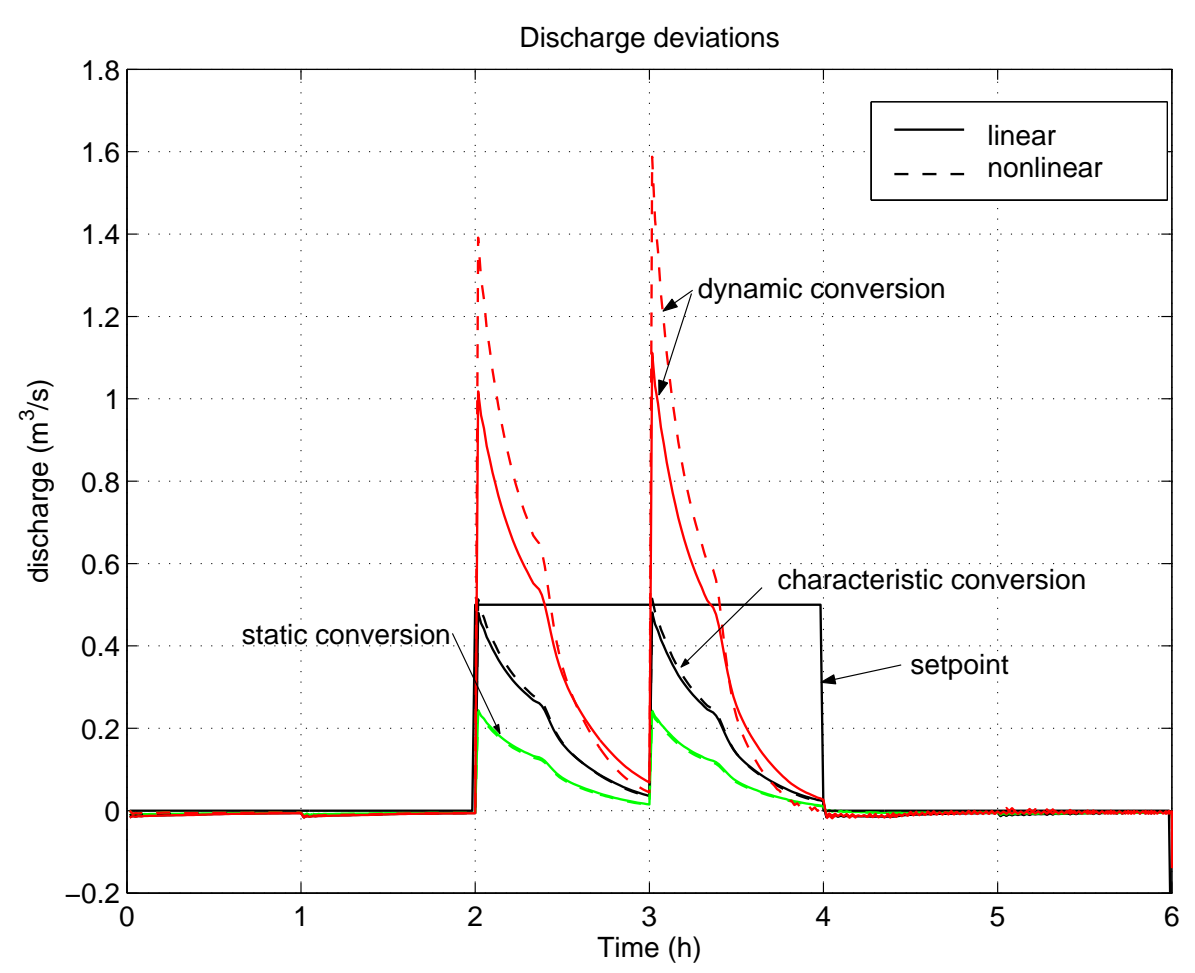

Figure 5: Discharge deviations for set-point following. Comparison of the linear and nonlinear versions of dynamic, characteristic and static conversion methods for $T_{s}=3600 \mathrm{~s}$.

\section{Experimental Results}

The experiments are performed on the Gignac Canal, located $40 \mathrm{~km}$ North-West of Montpellier, in the South of France.

\section{Description of Gignac Canal}

The main canal is $50 \mathrm{~km}$ long, with a feeder canal ( $8 \mathrm{~km}$ long) and two branches on the left and right banks of the Hérault river (resp. 27 and $15 \mathrm{~km} \mathrm{long).} \mathrm{The} \mathrm{canal} \mathrm{is} \mathrm{concrete} \mathrm{lined,} \mathrm{with}$ trapezoidal cross sections on the two branches, with average slopes of respectively, 0.00035 and $0.00050 \mathrm{~m} / \mathrm{m}$. The design flows are $3.5 \mathrm{~m}^{3} \mathrm{~s}^{-1}$ for the common trunk, $2.0 \mathrm{~m}^{3} \mathrm{~s}^{-1}$ for the left bank and $1.5 \mathrm{~m}^{3} \mathrm{~s}^{-1}$ for the right bank. The canal has been equipped with sensors, actuators 
and a SCADA system interfaced with the SIC-SCADA real-time module of the SIC software, which enables the monitoring and control of the cross-regulators of the right bank main canal.

\section{Experimental Setup}

The SCADA equipment has been designed to enable real-time monitoring and control of the canal, through a radio communication network and a real-time SCADA interface. The measured data is stored in a database, localized in the central control station.

The system has been configured to enable real-time control via the SCADA interface of SIC software. This interface, already presented in Litrico et al. (2007), enables real-time control of an irrigation canal using SIC software. This very generic method can easily be extended to work on any SCADA system.

The considered regulation gate is the gate at Avencq cross regulator, where water level sensors are installed upstream and downstream of the gate. An ultrasonic velocity sensor is also present a few meters downstream of the gate, which enables to compute the discharge flowing through the gate with a good accuracy. This measurement will only be used as a reference for evaluating the inversion methods.

\section{Experimental Results}

A scenario similar to the one used in simulation is tested at Avencq regulation gate. This gate is 1 meter large, with a discharge coefficient of 0.465 .

We compared the three methods, in their linear and nonlinear versions, for a sampling time step $T_{s}=15 \mathrm{~min}$. The discharge flowing through the gate is measured independently with an acoustic velocimeter located a few meter downstream of the gate. This sensor is able to deliver an accurate discharge measurement every minute. This discharge measurement is only used to validate the various methods. The measurements are done every minute, but only the water levels measured at the sampling time instants (every $T_{s}$ ) are used to compute the gate opening.

The initial discharge was around $0.32 \mathrm{~m}^{3} / \mathrm{s}$, and we tested the nonlinear static, characteristics and dynamic conversion methods, requiring an increase of $0.15 \mathrm{~m}^{3} / \mathrm{s}$, in order to deliver a discharge equal to $0.47 \mathrm{~m}^{3} / \mathrm{s}$ during 30 minutes. The results are displayed in Fig. 6 .

The results are very similar to the ones obtained in simulation (Cf. Fig. 5). The static method gives the worse results, since only $53 \%$ of the desired volume is delivered by the gate. The characteristics method is better, but the total volume is still only $79 \%$ of the desired one. The dynamic method delivers $97 \%$ of the desired volume.

The experimental results therefore validate the interest of using a dynamic approximation to compute the gate opening in order to deliver a desired discharge at a gate.

\section{Discussion}

The simulation and experimental results show the interest of developing a dynamic method. Indeed, for large sampling time steps, the classical static method leads to a very low performance. The characteristics methods improves the conversion, but also tends to underestimate the opening to deliver a given discharge for larger sampling time steps.

Such conversion methods (static, characteristics or dynamic) may be viewed as proportional controllers for the dynamical system relating the gate opening to the discharge flowing through the gate. Being calculated at each sampling period $T_{s}$ in incremental form (a discharge deviation generates a gate opening variation), all methods would converge asymptotically towards the good solution with zero steady state error. But, at each previous instant, the error magnitude 


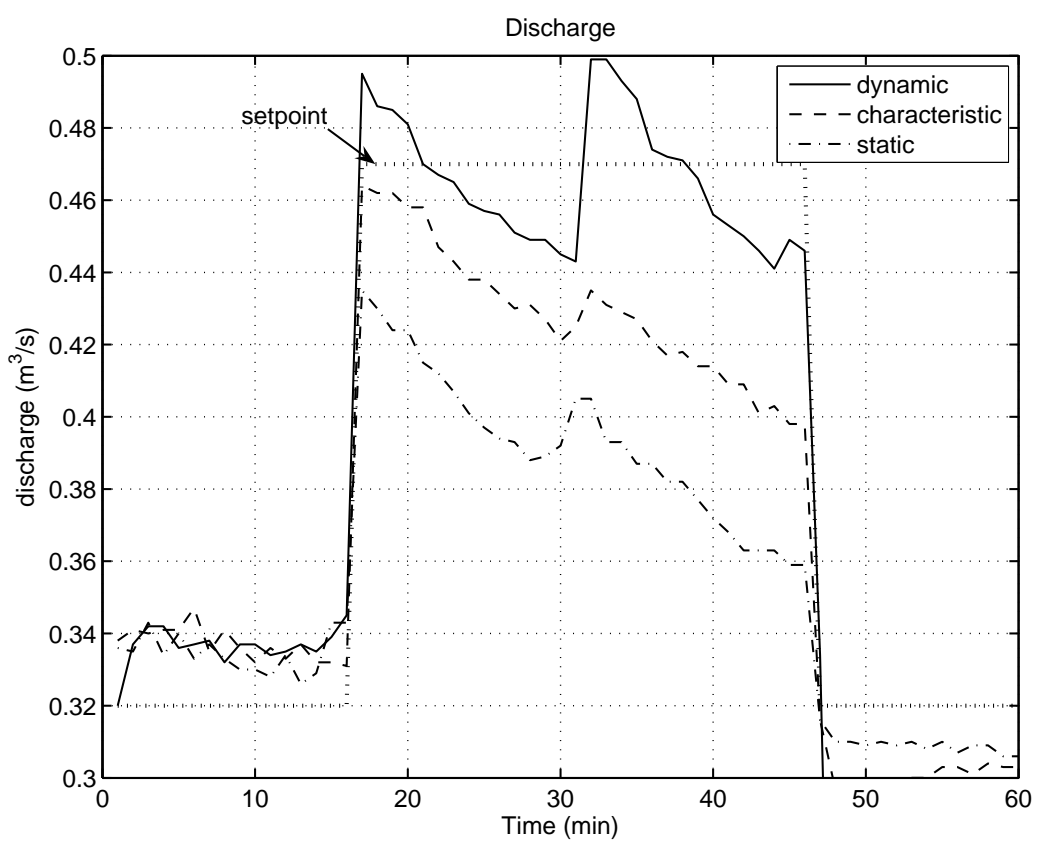

Figure 6: Experimental results: comparison of the delivered discharge at Avencq regulation gate for the nonlinear version of dynamic, characteristic and static conversion methods for $T_{s}=15 \mathrm{~min}$.

depends on the gain of the proportional controller. This gain (obtained from the relation between the gate opening and the discharge) is better calculated in the case of the dynamic method than for the characteristic method and for the static method. This explains why the dynamic method behavior is better than the two others.

When such a conversion method is used in conjunction with a master controller, the error in the delivered discharge may be compensated by the master controller. However, this discharge error will affect the overall performance of the controlled system. The better the slave controller performance, the closer the performance of the overall system will be to the performance of the master controller.

Therefore, we emphasize the fact that using an accurate conversion method such as the one proposed in this paper for the slave controller may improve the performance of master controllers using the discharges at regulation gates as control inputs. It is also possible to use more complex dynamical slave controllers, but the stability analysis of the master-slave structure should then be carefully studied.

\section{Dimensionless Analysis of the Linear Conversion Methods}

We now examine the domain of applicability of the static and characteristics linear methods, taking the linear dynamic method as a reference. As we have seen above, the dynamic method provides a very good approximation for the discharge flowing through a gate, and the static and characteristics methods lead to poor approximations, especially for large sampling times. However, this result depends on the physical characteristics of each pool, of the gate, and of 
the value of the sampling time.

In the following, we develop a dimensionless approach to evaluate the volume error between the static method (or the characteristics method) and the dynamic one. This leads to generic results that apply for any pool and gate, in order to decide which method should be used, accepting a certain error level.

\section{Dimensionless Formulation}

We define the reference time as $T_{r}=a / b$, with $a$ and $b$ given by Eqs. (16-17), and the reference discharge as $q_{r}=k_{w} w_{0}$. Now, with the dimensionless time $t^{*}=t / T_{r}$, we get the dimensionless discharge estimated by the three linear methods:

$$
\begin{aligned}
q_{s}^{l *}\left(t^{*}\right) & =1 \\
q_{c}^{l *}\left(t^{*}\right) & =\frac{1}{a} \\
q_{d}^{l *}\left(t^{*}\right) & =\frac{e^{-t^{*}}}{a}
\end{aligned}
$$

According to these equations and Eq. (35), the volume flowing through the gate during $T_{s}^{*}=T_{s} / T_{r}$ after a unit step gate operation is approximated by:

$$
\begin{aligned}
V_{s}^{*}\left(T_{s}^{*}\right) & =T_{s}^{*} \\
V_{c}^{*}\left(T_{s}^{*}\right) & =\frac{T_{s}^{*}}{a} \\
V_{d}^{*}\left(T_{s}^{*}\right) & =\frac{1-e^{-T_{s}^{*}}}{a}
\end{aligned}
$$

Let us note that these expressions only depend on the dimensionless parameter $a$ and the dimensionless sampling time $T_{s}^{*}$.

Now, we use the volume given by the dynamic method as a reference, and compute the volume error when using the static or the characteristics method. Then, the volume errors are defined as:

$$
\begin{aligned}
& e_{s}^{*}\left(T_{s}^{*}\right)=100\left(\frac{V_{s}^{*}}{V_{d}^{*}}-1\right) \\
& e_{c}^{*}\left(T_{s}^{*}\right)=100\left(\frac{V_{c}^{*}}{V_{d}^{*}}-1\right)
\end{aligned}
$$

These equations enable us to evaluate the volume error of the static or the characteristics method according to the dimensionless parameter $a$ and the dimensionless parameter $T_{s}^{*}$.

\section{Validity of Conversion Methods}

The volume error due to using the static or the characteristics method is now evaluated using the previous dimensionless expressions.

Figure 7 depicts the volume errors as a function of both dimensionless parameters $a$ and $T_{s}^{*}$. It appears that for a given $T_{s}^{*}$, the characteristics method leads to a constant volume error whatever the value of parameter $a$. On the contrary, the static method leads to an increasing error as parameter $a$ increases. For values of parameter $a$ larger than 1.5, the volume error with the static method is always larger than $50 \%$, even for small $T_{s}^{*}$. 


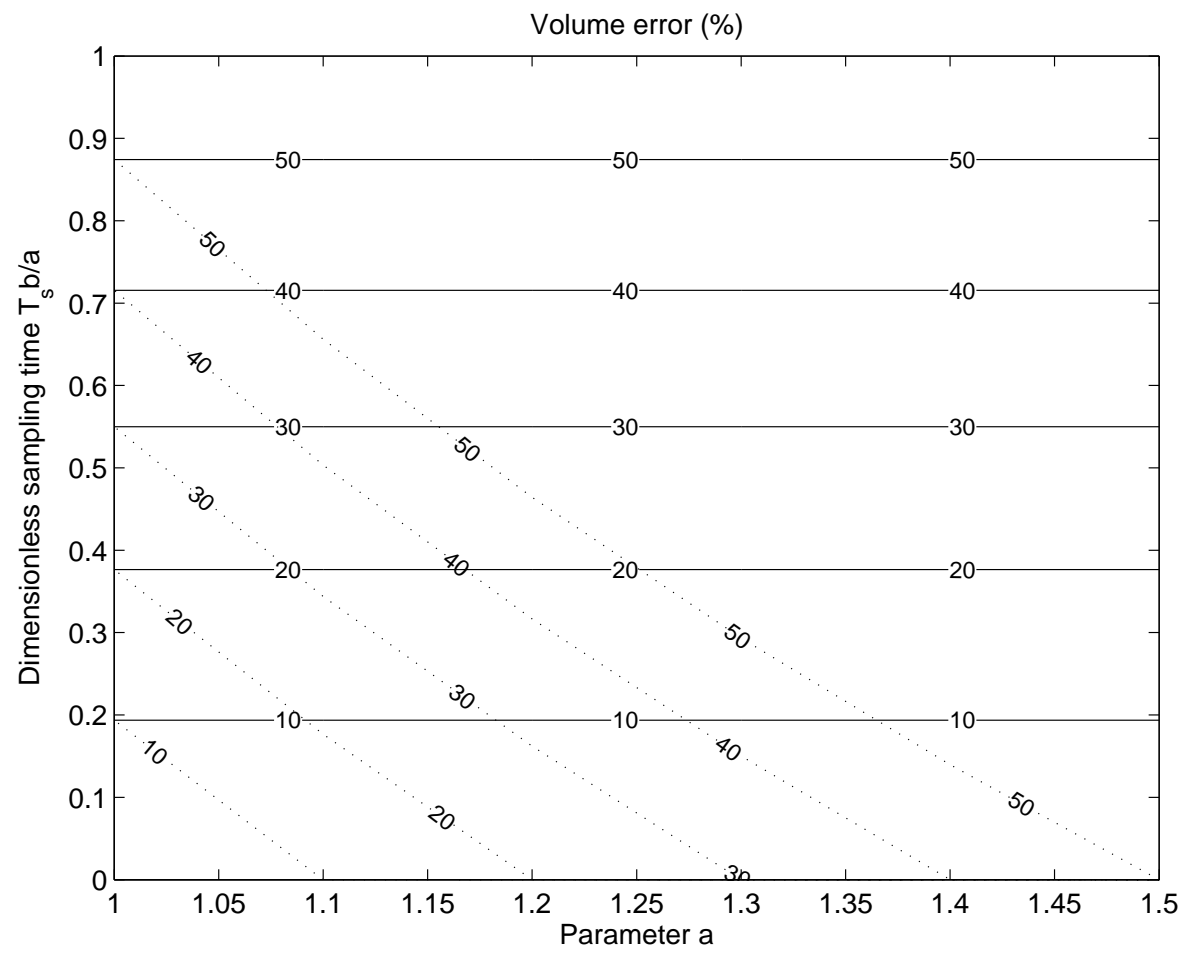

Figure 7: Volume error as a function of the dimensionless parameter $a$ and the dimensionless sampling time $T_{s} b / a$ : static method (dotted lines) and characteristics method (solid lines).

\section{Applicability of the methods}

It should also be mentioned that the data requirements are not the same for the different methods. In the static method, only the gate characteristics is needed. In the characteristics method, one also needs the channel local geometry. In the dynamic method, the complete channel geometry with the friction coefficient are needed.

Therefore, in a given application, one may use one or another method according to the available data.

\section{Conclusion}

The paper considered the problem of converting discharge into gate opening for the control of an open-channel equipped with regulation gates. We have reviewed the existing static and characteristics methods and we have proposed a new method that takes into account the dynamics of each pool. This dynamic method uses the IDZ model to get a closed form analytical solution for the discharge and the water levels deviations after a step gate opening or closing. It is then used to compute the necessary gate opening to deliver a desired average discharge during the sampling time step. Both a linear and a nonlinear version of each method has been developed.

The methods have been applied in simulation on an hypothetical canal, and in reality on a gate of the Gignac canal. The results have shown the performance of the dynamic method, which appears as a very effective means to determine the gate opening in order to deliver 
a desired discharge during a given sampling time period. Such a method can be used for automatic control, but also for manual control of an irrigation canal.

Finally, a dimensionless study has quantified the applicability of the static and characteristics methods compared to the dynamic one, which provides an effective tool to determine which method to use in practice.

\section{Appendix: Step Response of a First Order Linear Dynamic Sys- tem}

Let us compute the step response of a first order system, with transfer function:

$$
\hat{f}(s)=\frac{1}{s-p}
$$

and $p \neq 0$ is the pole of the system.

Impulse response The impulse response corresponds to the time response to an impulse input $\delta(0)$, with $\delta(t)$ the Dirac impulse. The time impulse response is given by the inverse Laplace transform of $\hat{f}(s)$ (see Abramowitz and Stegun (1972)):

$$
f(t)=e^{p t}
$$

Step response The step response is obtained as the inverse Laplace transform of $\hat{f}(s) / s$ :

$$
f(t)=\frac{1}{p}\left(e^{p t}-1\right)
$$

Response to non zero initial condition The effect of nonzero initial condition $f(0)$ is similar as the one of the impulse response:

$$
f(t)=f(0) e^{p t}
$$

Finally, using the linear superposition principle, these effects can be computed separately and combined to obtain the response of the dynamic system for different conditions.

\section{Acknowledgments}

The writers acknowledge the financial help of Région Languedoc-Roussillon, Conseil Général de l'Hérault, Agence de l'Eau Rhône-Méditerranée-Corse and Cemagref through the "Plateforme expérimentale du canal de Gignac". We thank Vincent Kulesza, head of the ASA de Gignac, for his collaboration in the experiments.

\section{Notations}

The following symbols are used in this paper:

$*=$ superscript for dimensionless values;

$\alpha_{i}=$ parameter linked to the characteristics of pool $i$;

$a=$ dimensionless parameter; 
$b=$ parameter in $s^{-1}$;

$a_{i}=$ high frequency gain of IDZ model for pool $i$ in sm ${ }^{-2}$;

$b_{i}=$ integrator gain of IDZ model for pool $i$ in $\mathrm{m}^{-2}$;

$C_{d}=$ discharge coefficient;

$C_{i}=$ celerity in pool $i$ in $\mathrm{ms}^{-1}$;

$e_{s, c, d}=$ relative volume error in $\%$ for static, characteristics and dynamic method, respectively;

$f=$ nonlinear function for the gate equation;

$g=$ gravitational acceleration;

$j=$ complex number $j^{2}=-1$;

$k_{u}, k_{d}, k_{w}=$ linearized gate coefficients in $\mathrm{m}^{2} \mathrm{~s}^{-1}$;

$L_{g}=$ gate width in $\mathrm{m}$;

$g_{i}(s)=$ Saint-Venant transfer functions for pool $i$;

$p=$ pole of the system in $\mathrm{s}^{-1}$;

$Q_{0}=$ steady state discharge in $\mathrm{m}^{3} \mathrm{~s}^{-1}$;

$q=$ discharge deviation in $\mathrm{m}^{3} \mathrm{~s}^{-1}$;

$q_{s}^{l}, q_{c}^{l}, q_{d}^{l}=$ discharge deviations with the linear version of static, characteristic and dynamic methods, respectively, in $\mathrm{m}^{3} \mathrm{~s}^{-1}$;

$q_{s}^{n l}, q_{c}^{n l}, q_{d}^{n l}=$ discharge deviations with the nonlinear version of static, characteristic and dynamic methods, respectively, in $\mathrm{m}^{3} \mathrm{~s}^{-1}$;

$\bar{q}=$ average discharge deviation in $\mathrm{m}^{3} \mathrm{~s}^{-1}$

$\bar{q}_{\text {max }}=$ maximum average discharge deviation in $\mathrm{m}^{3} \mathrm{~s}^{-1}$;

$s=$ Laplace variable in $\mathrm{s}^{-1}$;

$T_{i}=$ top width in pool $i$ in $\mathrm{m}$;

$T_{m}=$ time period in $\mathrm{s}$;

$T_{r}=$ reference time in s;

$T_{s}=$ sampling time in $\mathrm{s}$;

$t=$ time in $\mathrm{s}$;

$V_{i}=$ water velocity in pool $i$ in $\mathrm{m}$;

$V_{s}, V_{c}, V_{d}=$ volume deviations with static, characteristic and dynamic method, respectively, in $\mathrm{m}^{3}$;

$W=$ absolute gate opening in $\mathrm{m}$;

$w=$ gate opening deviation in $\mathrm{m}$;

$w_{\max }=$ maximum gate opening deviation in $\mathrm{m}$;

$w_{s}^{l}, w_{c}^{l}, w_{d}^{l}=$ gate opening deviations for the linear version of static, characteristic and dynamic method, respectively, in m;

$w_{s}^{n l}, w_{c}^{n l}, w_{d}^{n l}=$ gate opening deviations for the nonlinear version of static, characteristic and dynamic method, respectively, in m;

$X_{i}=$ length of pool $i$ in $\mathrm{m}$;

$Y_{i}=$ steady state water depth in $\mathrm{m}$ for pool $i$;

$y_{i}=$ water level deviation in $\mathrm{m}$ for pool $i$;

\section{References}

Abramowitz, M. and Stegun, I. A. (1972). Handbook of mathematical functions, volume 55 of Applied Mathematics Series. National Bureau of Standards, 10th edition.

Baume, J.-P. and Sau, J. (1997). Study of irrigation canal dynamics for control purposes. 
In Int. Workshop on the Regulation of Irrigation Canals, RIC'97, pages 3-12, Marrakech, Morroco.

De Leon Mojarro, B. (1986). Contribution à l'amélioration de la gestion des périmètres irrigués. Ph.D. thesis, USTL Montpellier. 137 p.

Litrico, X. and Fromion, V. (2004a). Frequency modeling of open channel flow. J. Hydraul. Eng., 130(8):806-815.

Litrico, X. and Fromion, V. (2004b). Simplified modelling of irrigation canals for controller design. J. Irrig. Drain. Eng., 130(5):373-383.

Litrico, X., Malaterre, P.-O., Baume, J.-P., Vion, P.-Y., and Ribot-Bruno, J. (2007). Automatic tuning of PI controllers for an irrigation canal pool. J. Irrig. Drain. Eng., 133(1):27-37.

Malaterre, P.-O. (1995). Regulation of irrigation canals: Characterisation and classification. Irrigation and Drainage Systems, 9(4):297-327.

Malaterre, P.-O. (2006). SIC 4.20, Simulation of Irrigation Canals. http://sic.malaterre.net/sic/sicgb.htm.

Malaterre, P.-O. and Baume, J.-P. (1999). Optimum choice of control action variables and linked algorithms. Comparison of different alternatives. In Workshop on Modernization of Irrigation Water Delivery Systems, pages 387-406, Phoenix, Arizona, USA.

Malaterre, P.-O., Rogers, D. C., and Schuurmans, J. (1998). Classification of canal control algorithms. J. Irrig. Drain. Eng., 124(1):3-10.

Schuurmans, J. (1997). Control of water levels in open-channels. Ph.D. thesis, ISBN 909010995-1, Delft University of Technology. 